Journal of Computer Science 8 (1): 55-60, 2012

ISSN 1549-3636

(C) 2012 Science Publications

\title{
Energy Efficient Aggregation for Continuous Monitoring Applications of Wireless Sensor Network
}

\author{
${ }^{1}$ R. Rajkamal and ${ }^{2}$ P. Vanaja Ranjan \\ ${ }^{1}$ Department of Electronics and Communication Engineering, \\ Alpha College of Engineering, Thirumazhisai, Chennai-600 124, Tamil Nadu, India, \\ ${ }^{2}$ Department of Electrical and Electronics Engineering, \\ Division of Embedded System Technology, College of Engineering Guindy, \\ Anna University Chennai-25, Tamil Nadu, India
}

\begin{abstract}
Problem statement: Continuous Monitoring of time critical phenomenon is one of the major streams of possible application in this arena. Sensing and Monitoring of such phenomenon involves in dealing with large amount of data correlated in spatial and temporal dimensions. Most applications of WSN, the nodes are hard to reach and it is impossible to replace their batteries so the nodes must operate without battery replacement for a long time. Approach: Aggregation techniques plays major role in increasing network life time by reducing the amount of data and data transmission in the resource limited (battery) Wireless Sensor Network (WSN). By exploring the impact of heterogeneity on the data aggregation protocols, energy consumption of radio of the WSN is significantly reduced. Results: In this study, we proposed a network model and an aggregation scheme for Continuous monitoring time critical applications. We have presented the simulation results that rationalize the proposed scheme. The comparison is made with LEACH, HEED, CLUDDA and TEEN in terms of energy dissipation showing promising improvement by applying the proposed scheme. Conclusion: The proposed scheme is well suited for continuous monitoring of time critical applications and also showing promising improvement in terms of network life time.
\end{abstract}

Key words: Continuous monitoring, time critical applications, hierarchical network, network life time, data aggregation, energy dissipation, showing promising, proposed scheme, wireless sensor network, large amount

\section{INTRODUCTION}

Wireless Sensor Network (WSN) is a system that relies on the collective effort of several micro nodes that promise fine-grain monitoring in wide variety of environments. The ability to communicate not only allows the network of nodes to communicate information and control across it but also nodes to cooperate in performing more complex tasks like statistical sampling, data aggregation (Intanagonwitat et al., 2002), system health and status monitoring.

A Sensor node (generally known as mote) is made up of four basic components namely sensing unit, processing unit, transceiver and power unit. Sensing units are usually composed of two sub units: one or more number of sensors and Analog-to-Digital Converters (ADCs). The processing unit is generally associated with a small storage unit, manages the procedures that make the sensor node collaborate with the other nodes to carry out the assigned sensing tasks. The transceiver unit connects the node to the network. The sensor node is being a micro electronic device; the power unit is treated as most important unit (Fakildiz et al., 2002). Therefore, sensor node lifetime shows a strong dependence on battery lifetime.

The energy consumption of a sensor node occurs in three domains namely Sensing, Data Processing and Communication. The dominant energy consumption in the WSN occurs in the radio transceivers (i.e., Communication domain of a sensor node) (Min et al., 2002; Sohrabi, 2000; Estrin, 1999; Raghunathan, 2002). Comparatively the energy consumption of the other components in the sensor node is very small. For this reason, many researches address this issue in WSN and advanced to reduce the wireless transmission. Data aggregation has been put forward as an essential paradigm in communication domain of sensor networks. The idea behind the data aggregation is to combine the data coming from different sources, enrooted-eliminating redundancy, minimizing the

Corresponding Author: R. Rajkamal, Department of Electronics and Communication Engineering, Alpha College of Engineering, Thirumazhisai, Chennai-600 124, Tamil Nadu, India 
number of transmissions and thus saving energy. The main impetus of study lies in increase the network life time of WSN.

Preliminaries: The most important aspect of WSN is an efficient use of energy in network environment and there are efficient routing and MAC protocols being introduced. The design of efficient data aggregation algorithm is an inherently challenging task. There has been intense research in the recent past on data aggregation algorithms of WSNs. In hierarchical structure of WSN, although data - aggregation is energy efficient and in - network processing is easy, there are problems that the overhead in the hierarchical structure is large and needs to be created again where the mobility of the nodes is concern, because the use of energy is focused on nodes like cluster head. In addition to that all sensor nodes are not used in the communication and a large amount of data processing is carried out in cluster head node. In a heterogeneous network, two or more different types of nodes with different battery energy and functionality are used. The data aggregation protocol of hierarchical networks is classified based on Cluster-Based networks, ChainBased networks, Tree-Based networks and Grid-Based networks. We describe the cluster based data aggregation protocols in the rest of the section, since this research work is concentrated on Cluster-Based network. Recently, several cluster-based network organization and data-aggregation protocols have been proposed. we discuss four such protocols, namely, Low-Energy Adaptive Clustering Hierarchy (LEACH) (Heinzelman, 2000), the Hybrid Energy-Efficient Distributed clustering approach (HEED) (Younis and Fahmy, 2004), Clustered Diffusion with Dynamic Data Aggregation (CLUDDA) (Chatterjea and Havinga, 2003) and threshold Sensitive Energy Efficient Sensor Network Protocol (TEEN).

Heinzelman, (2000), were the first to propose energy conserving cluster formation protocol called LEACH. The two main phases involved in LEACH are the set-up phase and the steady-state phase. The set-up phase involves the organization of the network into clusters and the selection of cluster heads. The steadystate phase involves data aggregation at the cluster heads and data transmission to the sink. LEACH was compared with Minimum Transmission Energy (MTE) routing in which intermediate nodes are chosen such that the sum of squared distances between adjacent nodes of the route is minimized. Although LEACH improves the system lifetime and data accuracy of the network, the protocol has the following limitations. LEACH assumes that all sensors have enough power to reach the sink if needed. In other words, each sensor has the capability to act as a cluster head and perform data fusion. This assumption might not be valid with energy -constrained sensors. LEACH also assumes that nodes have data to send periodically. In LEACH, all nodes have the equal amount of energy capacity in each election round, which is based on the assumption that being a cluster head results in same energy consumption for every node. Hence, LEACH should be extended to account for node heterogeneity.

Younis and Fahmy, (2004) have proposed HEED, whose main goal is to form efficient clusters for maximizing network's lifetime. The main assumption in HEED is the availability of multiple power levels at sensor nodes. Inter-cluster communication has not been considered in HEED. The performance of HEED has been compared with generalized LEACH (genLEACH) proposed in (Younis and Fahmy, 2004). The protocols were simulated for different sizes of network. The simulation results reveal that HEED improves the network's lifetime over gen-LEACH. In gen-LEACH the selection of cluster heads is random, which may result in rapid death of certain nodes. However, in HEED the cluster heads are selected such that they are well distributed with minimum communication cost.

The new data aggregation scheme proposed in (Chatterjea and Havinga, 2003) is called Clustered Diffusion with Dynamic Data Aggregation (CLUDDA) which combines clustering with diffusion mechanisms. CLUDDA performs data aggregation in unfamiliar environments by including query definitions within interest messages. CLUDDA combines Directed Diffusion (Intanagonwiwat et al., 2003) with clustering during the initial phase of interest or query propagation. The clustering approach ensures that only cluster heads and gateway nodes which perform inter cluster communication are involved in the transmission of interest messages. This technique conserves energy, since the regular nodes remain silent unless they are capable of servicing a request. In CLUDDA, the aggregation points are dynamic. The dataaggregation task is not assigned to any specific group of nodes in the network.

In (Manjeshwar and Agarwal, 2001), TEEN is proposed as an event driven data generation approach where energy is saved by limiting generation of the packets. To limit the number of packets, the protocol introduces two thresholds of sensed value namely a hard threshold and a soft threshold. Data is only sent to sink if the current value is greater than hard threshold or if the difference between the current and previous value is greater or equal to soft threshold. Most of the other existing work like EADAT (Ding et al., 2003), PEGASIS (Lindsey and Raghavendra, 2002), PEDAP 
(Tan and Korpeoglu, 2003), SAG (Azim et al., 2010) and adaptive modulation for QoS with energy consumption analysis (Yuan et al., 2005) has mainly focused on the communication domain, especially in the aspects of routing, Medium Access Control (MAC) and collaborative mechanism for data aggregation of other categories of hierarchical network like Chain-Based networks, Tree-Based networks and Grid-Based networks.

From the above discussion it is clear that the performance of the data aggregation protocol is strongly coupled with the infrastructure of the network. There has not been significant research on exploring the impact of heterogeneity on the performance of the data aggregation protocols. This motivates us to explore the impact of heterogeneity of the WSN for continuous monitoring time critical applications such as intrusion detection, electric Power Quality monitoring.

\section{MATERIALS AND METHODS}

A lot of sensor nodes are dispersed on an interested region. Base Station or Sink nodes are placed at convenient places in or near the environment to be monitored. The users can monitor the information from the sensor field through the sink nodes by direct or remote access to it. Thus the sink node should have user interface or capabilities to communicate with remote users using high powered radio or wired connection.

The desired sensing area (area to be monitored) is considered as square with an area of $\mathrm{A}$. The region $\mathrm{A}$ is divided into number of equal squares based on the number of Cluster Head $(\mathrm{CH})$ nodes. The center of each square is computed by Eq. 1 and $\mathrm{CHs}$ are placed in center point of each square:

Center of the square $\mathrm{C}=0.707 * \mathrm{a}$

$\mathrm{C}$ should be measured on the diagonal of the square or $\mathrm{C}$ can be calculated in $\mathrm{X}-\mathrm{Y}$ coordinates (i.e., the center of the square can be located as $(\mathrm{a} / 2, \mathrm{a} / 2)$.

The sensing range depends on the emission power of the sensing signal, the propagation loss and the sensing sensitivity. The sensing sensitivity of each $\mathrm{CH}$ is assumed to be $\Omega_{\text {sen }}$ (in decibel units); i.e., the received signal power must be larger than $\Omega_{\text {sen }}$ for a source being sensed by a node for successful detection of sensing signal. In addition, the sensing signal power emitted by a target source is assumed to be $\Omega_{\mathrm{t}}$ (in decibel units). It is assumed all nodes in the network are having omni-directional antennas with maximum transmit power of $\mathrm{P}_{\max }$. Further study is not dealt with coverage problems of the WSN.

Only few number of sensor nodes that have special capability, battery with larger capacity or external power supply are called $\mathrm{CH}$. All CHs are assumed to be homogeneous, i.e. with the same ability and sensitivity. All the other sensor nodes have the same constant transmission ranges.

The distribution of the sensor nodes is assumed uniformly random within this area and the deployment is independent for different nodes, i.e., the probabilities of placing the sensor nodes onto any locations in the area are the same. The number of the sensor nodes $\mathrm{N}(\mathrm{A})$ in the area A with intensity $\lambda$ is determined by the homogeneous Poisson process is given in Eq. 2:

$$
\begin{aligned}
\mathrm{P}[\mathrm{N}(\mathrm{A})=\mathrm{x}] & =\frac{\mathrm{e}^{\mathrm{x} \text { area }} *[\lambda * \operatorname{area}(\mathrm{A})]^{*}}{\mathrm{x} !} \\
\mathrm{x} & =0,1,2
\end{aligned}
$$

$\mathrm{N}(\mathrm{A}) \quad=$ Number of nodes

$\lambda=$ Expected number of nodes per unit time (Intensity)

$\lambda *$ area $(\mathrm{A})=$ Mean number of nodes over the area $\mathrm{A}$

Aggregation algorithm and characteristics of nodes: The motivation being that the more complex hardware and extra battery energy can be embedded in few Cluster Head $(\mathrm{CH})$ nodes, thereby reducing hardware cost of the rest of the network. This impact of the heterogeneous networks enabled us to design the network for continuous monitoring applications with a base station (sink-type 0), clusters having Cluster Heads (CH) (type-1) and number of Sensor Nodes (type-2) with the following capabilities.

Cluster head node (type-1): Receive/ Transmit raw data or queries from/to type-2 nodes in the cluster.

Transmit/ Receive raw data / information from / to type1 nodes of one cluster to other clusters in the network.

Ability to classify the events from received data.

Decision making capability to send /not to send the information/data to sink (type-0).

Sensor node (type-2): Transmit raw data to type-1 nodes in the cluster. Receive queries from type-1 node in the cluster. Ability to compare the sensed data with the stored data.

The main task on the motes in WSN monitoring applications is to carry out wireless communication 
through the radio transceiver. Generally the power consumption measurement for radio is considered the following different radio operation: receive (RX)/listen, Transmit (TX) under different transmission power thresholds, sleep and idle state. When a radio is in sleep or idle mode, its RF power rating is irrelevant. The RF power affects power consumption only when the radio is TX / RX mode.

By restricting the unnecessary data transmission in the continuous monitoring environment, the considerable energy saving in the radio transceiver section of type- 1 and type- 2 nodes are achieved through the following algorithm.

\section{Algorithm in type-2 node:}

1. Start timer

2. If (timer expired)

3. Start to sense the environment (for PQ disturbance)

4. Compute Mean (M), Standard Deviation (SD) and Skew (Sk) of the present sensed data (D1)

5. Compare with $\mathrm{M}, \mathrm{SD}, \mathrm{Sk}$ of the sensed data with the stored data's (D0) M, SD, Sk.

6. If (D1 not equal to D0)

7. Sensor nodes transmit D1 to $\mathrm{CH}$

8. Else

9. No transmission

10. Go to STEP 1

In the above described algorithm the M, SD and Sk of the data samples are computed for the standard test signal (e.g., In Power Quality Monitoring applications the Power Quality Disturbance is determined with respect to the pure sinusoidal signal) and stored in the sensor node. The stored data is compared with the sensed signal's M, SD and Sk. If, both are equal then it is considered as no event occurs.

\section{Algorithm in type-1 node:}

1. If (Node receives data)

2. Classify the data according to the nature of the event present in the data

3. Disturbance event information (not data) will be sent to sink

4. Else

5. Do nothing

6. If (request from base station to send the data)

7. Send data to base station

8. Else

9. Do nothing
The type-1 node classifies the received data according to the nature of the event occurs (e.g., in power quality monitoring applications disturbances are categorized according to the nature of disturbance in the data like sag, swell). Each event is assigned to different communication channel of the node. Zigbee standard IEEE 802.15.4 specifies 16 channels within the $2.4 \mathrm{GHz}$ band, in $5 \mathrm{MHz}$ steps, numbered 11 through 26 . The radio frequency of channel $\mathrm{k}$ is given by $\mathrm{Fc}=2405+5(\mathrm{k}-11) \mathrm{MHz}, \mathrm{k}=$ $11,12, \ldots 26$. For operation in channel $\mathrm{k}$, the FSCTRL.FREQ, 10-bit register should therefore be set to FSCTRL.FREQ $=357+5 \quad(\mathrm{k}-11)$. The classification of the data can be carried out by Neural Network Classifier. Once classification process is completed an empty packet will be sent in the form of carrier frequency to the base station in the specified kth channel. Since there are 16 channels supported by Zigbee standard, 16 different events can be monitored by the network.

With our protocol every cluster head that has a data /information ready to be transmitted would select a route to relay the packet to the Base Station. The route is chosen according to Minimum Transmission Energy (MTE) routing algorithm. Therefore, the algorithm chooses one or more intermediate cluster head nodes so that the sum of squared distances is minimized.

\section{RESULTS}

Simulation setup: We evaluate performance of well known hierarchical network protocols such as LEACH, HEED, CLUDDA and TEEN and are compared with the proposed technique in detail including energy efficiency and network lifetime. The performance is evaluated through NS2. The network is deployed in an area of $500 \times 500 \mathrm{~m}$. We have varied the number of nodes in each cluster as $20,40 \ldots .100$. The base station is assumed to be situated $100 \mathrm{~m}$ away from the above specified area. The simulated traffic is CBR with UDP. Table 1 summarizes the simulation parameters considered.

In this study, we have considered the hierarchical WSN for continuous monitoring based on event driven. The operation of LEACH, HEED and CLUDDA is broken up into rounds, where each round begins with a set-up phase when the clusters are organized, followed by a steady-state phase when data transfers to the base station occur. 


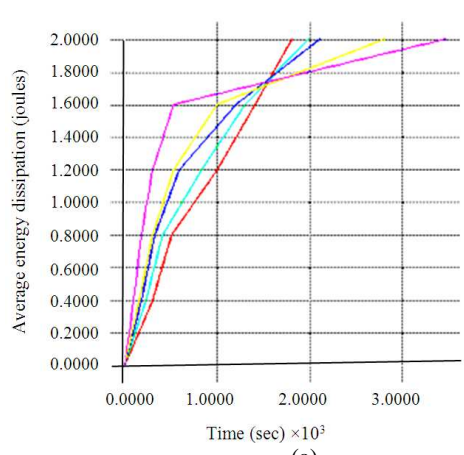

(a)

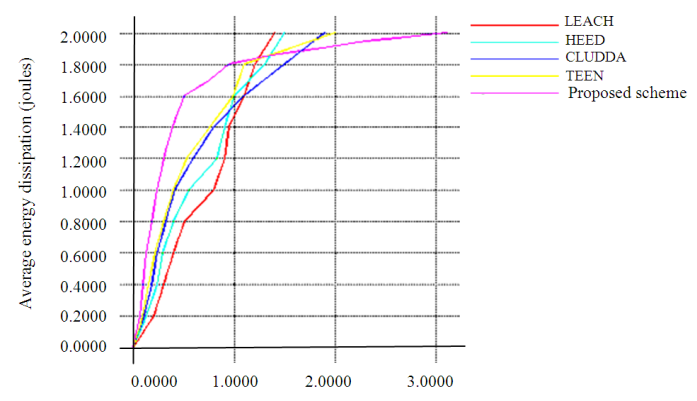

(c)

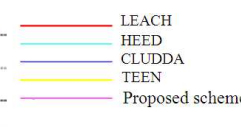

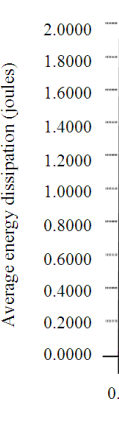

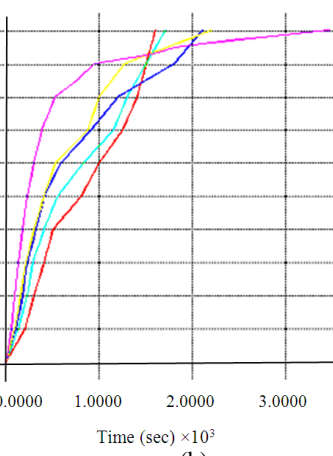

(b)

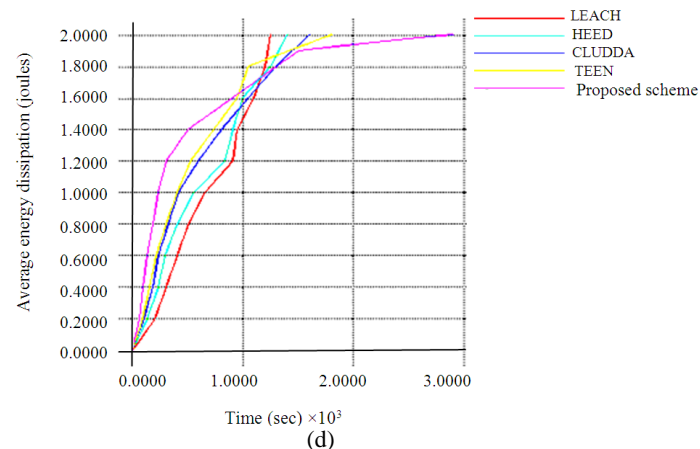

(d)

Fig. 1:Simulated results -70 nodes in each cluster. Average energy dissipation Vs time (a) number of events/ clusters $=20$ (b) number of events/ clusters $=30$ (c) number of events/ clusters $=40$ (d) number of events/ clusters $=50$

Table 1: Simulation parameters

\begin{tabular}{ll}
\hline Parameters & Value \\
\hline Area size & $500 \times 500 \mathrm{~m}$ \\
Area of each cluster & $125 \times 125 \mathrm{~m}$ \\
Number of clusters and clusters heads & 16 and 16 \\
No. of nodes in each cluster & 70 \\
MAC & IEEE 802.15 .4 \\
Simulation time & $3600 \mathrm{sec}$ \\
Traffic source & CBR \\
Packet size & 512 \\
Transmission range cluster head & $180 \mathrm{~m}$ \\
Transmission range of sensor node & $90 \mathrm{~m}$ \\
\hline
\end{tabular}

Table 2: Energy model

\begin{tabular}{ll}
\hline Parameters & Value \\
\hline Carrier frequency & $2.4 \mathrm{GHz}$ \\
Transmitter antenna gain & $1 \mathrm{~dB}$ \\
Receiver antenna gain & $1 \mathrm{~dB}$ \\
Antenna height & $1.5 \mathrm{~m}$ \\
Receive threshold & $2 \mathrm{nW}$ \\
Success threshold & $6 \mathrm{nW}$ \\
Initial energy of each node & $2 \mathrm{~J}$ \\
Bandwidth & $2 \mathrm{Mbps}$ \\
\hline
\end{tabular}

We have executed 5 runs of the simulator for each protocol with 20 events in each cluster $(20 * 16=320$ events). The number of nodes in each cluster is $70\left(70^{*}\right.$ $16=1120$ nodes in the network). The readings of 5 trails are averaged and plotted. Similarly it is repeated and plotted for 30, 40 and 50 events. The energy model of the protocols simulated is summarized in Table 2. Figure 1 show the performance of the protocols with the proposed network when 20,30, 40 and 50 event occurs in each cluster. It can be observed from the simulation results, the proposed scheme is showing promising improvement in network life time for more number of events.

\section{DISCUSSION}

In the proposed model the network structure and the cluster head is fixed. Hence the cluster organization in set-up phase is not playing any role in the application under consideration. For this kind of monitoring applications, only the data aggregation and communication $(\mathrm{Tx} / \mathrm{Rx})$ modules of the hierarchical protocols are considered for performance evaluation.

However, in set-up phase, there is no guarantee that nodes selected as cluster head are evenly dispersed throughout the network because procedure to select cluster head is based on the random cluster formation method having local probability. The protocol performs better when more number of nodes alive at any given time which shows the lower value of energy dissipation. The average energy dissipated shows the 
average dissipation of energy per node over the time in a network as it performs various functions such as transmitting, receiving, sensing, aggregation of data.

\section{CONCLUSION}

WSN have opened the doors to many applications that require monitoring and control. Designing a low power WSN will have a great benefit for most applications. In study we have proposed a cluster based network with an aggregation protocol by paying special attention on impact of heterogeneity (node's hardware). The proposed Scheme is well suited for time critical applications and is also showing promising improvement in terms of network life time.

In the proposed scheme, if the sensed data is equal to the stored data, the nodes will never communicate. The user will not get any data from the network. If the user wants to get the data, user has to issue query /request on the data required. Thus the scheme is not well suited for application where the user requires data on regular basis.

\section{REFERENCES}

Azim, M., A. Moad, S.N. Bouabdallah, 2010. SAG: Smart Aggregation Technique for ContinuousMonitoring in Wireless Sensor Networks. Proceeding of the IEEE Conference on Communications, May, 23-27, IEEE Xploor, Cape Town, pp: 1-6.

Chatterjea, S. and P. Havinga, 2003. A dynamic data aggregation scheme for wireless sensor networks. Proceeding of the 14th Workshop on Circuits, Systems and Signal Processing, Nov. 26-27, Veldhoven, Netherlands, pp: 1-7. http://purl.utwente.nl/publications/59890

Ding, M., X. Cheng and G. Xue, 2003. Aggregation tree construction in sensor networks. Proceeding of the Vehicular Technology Conference, Oct. 6-9, IEEE Xploor, USA., pp: 2168-2172. DOI: 10.1109/VETECF.2003.1285913

Estrin, D., 1999. Next century challenges: scalable coordination in sensor networks. Proceedings of the 5th Annual ACM/IEEE International Conference on Mobile Computing and Networking, ACM, New York, USA., pp: 263270. DOI: $10.1145 / 313451.313556$

Fakildiz, I., W. Su, Y. Sankarasubramaniam and E. Cayirci, 2002. A survey on sensor networks. IEEE Communi. Magazine, 40: 102-114. 10.1109/MCOM.2002.1024422
Heinzelman, W.R., 2000. An application-specific protocol architecture for wireless microsensor networks Wireless Commun. IEEE Trans., 1: 660671. DOI: 10.1109/TWC.2002.804190

Intanagonwitat, C., D. Estrin, R. Govindan and J. Heidemann, 2002. Impact of network density on data aggregation in wireless sensor networks. Proceeding of the 22nd International Conference on Distributed Computing Systems, (DCS' 02), IEEE Xploor, USA., pp: 457-458. DOI: 0.1109/ICDCS.2002.1022289

Intanagonwiwat, C., R. Govindan, D. Estrin, J. Heidemann and F. Silva, 2003. Directed diffusion for wireless sensor networking. ACM/IEEE Trans. Networking, $\quad 11$ : $2-16 . \quad$ DOI: 10.1109/TNET.2002.808417

Lindsey, S. and C. Raghavendra, 2002. PEGASIS: Power-efficient gathering in sensor information systems. Proceeding of the IEEE Aerospace Conference, Apr. 15, IEEE Xploor, USA., pp: 1113-1125. DOI: 10.1109/AERO.2002.1035242

Manjeshwar, A. and D.P. Agarwal, 2001. TEEN: A routing protocol for enhanced efficiency in wireless sensor networks. Proceeding of the IEEE Parallel and distributed Processing of the symposium, Apr. 2001, IEEE Xploor, USA., pp: 2009-2015. DOI: 10.1109/IPDPS.2001.925197

Min, R. et al., 2002. Energy-centric enabling technologies for wireless sensor networks. IEEE Wireless Commun., 9: 28-39. DOI: 10.1109/MWC.2002.1028875

Raghunathan, V., 2002. Energy-aware wireless microsensor networks. IEEE Signal Proc., 19: 4050. DOI: $10.1109 / 79.985679$

Sohrabi, K., 2000. Protocols for self-organization of a wireless sensor network. IEEE Personal Commun., 7: 16-27. DOI: 10.1109/98.878532 .

Tan, H.O. and I. Korpeoglu, 2003. Power efficient data gathering and aggregation in wireless sensor networks. SIGMOD Record, 32: 66-71. DOI: 10.1145/959060.959072

Younis, O. and S. Fahmy, 2004. HEED: A Hybrid, energy-efficient, distributed clustering approach for ad hoc sensor networks. IEEE Trans. Mobile Comput., 3: 366-379. DOI: 10.1109/TMC.2004.41

Yuan, Y., Z. Yang and J. He, 2005. An adaptive modulation scaling scheme for quality of services ensurance in wireless sensor networks. Am. J. Applied Sci., 2: 734-738. DOI: 10.3844/ajassp.2005.734.738. 